\title{
DEVELOPMENT OF A BOOLEAN LOGIC SIMULATOR BASED ON QUINE MCCLUSKY ALGORITHM
}

\author{
I. Oghogho ${ }^{1,}{ }^{*}$, H. Agada2 ${ }^{2}$ and A. M. Obiazi ${ }^{3}$ \\ 1, Department of Electrícal \& Electronic Engineeríng, Delta State University, Abraka-Oleh Campus, \\ DELTA STATE, NİGERİA \\ 2, DePT OF ElECTRÍCAL \& INFORMATION ENGR'G, LANDMARK UNIVERSITY, OMU-ARAN, KWARA STATE, NİGERİA

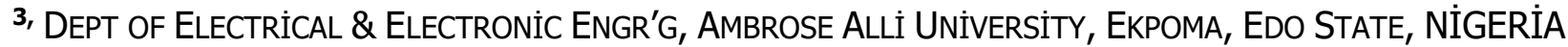 \\ E-mail addresses: ${ }^{1}$ oghogho.ikponmwosa@delsu.edu.ng, ${ }^{2}$ caleb.agada@lmu.edu.ng, \\ 3 biazimoz@gmail.com
}

\begin{abstract}
This paper presents the development of an educational simulation tool for solving Boolean logic equations using Quine McCluskey's algorithm. Unity3D development environment was used to provide an interface for the design of the software front end. A custom built version of Javascript was used to write the back end code that drives the algorithm. Based on the output parameters decided on by the user for either sum of product (SOP) or product of sum (POS) approaches, the software provides an interactive user interface displaying: (i) the truth table with a maximum of six inputs (ii) the Karnaugh Map as well as the generated corresponding simplified logic equation and (iii) the generated logic circuit diagram. The results of the simplified Boolean expressions and logic circuits generated by the developed tool were compared with manually calculated solutions and available htm/ and console based implementations and were found to be accurate.
\end{abstract}

Keywords: Boolean Logic Simulator, Logic Circuit, Quine McClusky Algorithm, Karnaugh Map, Digital Electronics.

\section{INTRODUCTION}

Digital Electronics covers Boolean logic expressions, their simplification, their representation in truth tables and how to plot functional logic circuit diagrams based on a desired predetermined result [1]. Further down the course, several methods of logic simplification for the reduction of more complex expressions are introduced. The process generally involves the dissolution of redundant components of the expressions with the use of Boolean arithmetic or graphical methods like Karnaugh maps. In many applications, it is required that a Boolean equation is solved while excluding irrelevant variables $[2,3]$.

The five key elements in Boolean logic simplification are:

(i) the truth table, which expresses output states based on all possible permutations of variable input states, (ii) the $\mathrm{min} / \max$ term, which is a simple way of describing the system's "ON" states, as expressed by the truth table

(iii) the Karnaugh map, which is a graphical method of simplifying Boolean equations,

(iv) the simplified Boolean expression, which is the shortest and most logical expression for the desired outputs with all permutations of inputs for the system, and

(v) the circuit diagram, which is the final logic circuit that satisfies any logic equation.

Digital circuit designers use Boolean algebra as the basic formal system while digital circuits are the end results they seek to produce. Typically, the complexity of the raw expressions derived from truth tables and in most cases, the final decomposed algorithms, increases exponentially with the number of variables

* Corresponding author, tel: $+234-806-067-6748$ 
in question. Because of this, the manual computation of systems with higher variable counts is extremely tedious and cumbersome even with the use of simplified methods like the Karnaugh map. Lecturers and students alike have a hard time simulating larger systems in class during teaching sessions due to time constraints. Examples in class only border around simplistic circuit designs with no more than four variable inputs and most students of the subject never get to fully understand the concepts of Karnaugh mapping, or grasp the correlation between the key elements in Boolean logic simplification for more than four variables.

It is therefore necessary to create a learning tool which assists both students and their teacher, to easily and effectively simulate simple and complex Boolean equations, and visualize the simplification using Karnaugh maps and the final logic circuit diagram. This will enable the visual assessment of the correlation between each of the elements mentioned above without the need for cumbersome manual calculation. A Boolean function simplification algorithm such as the Quine McCluskey algorithm [4] and a robust programming interface such as the Unity engine makes this possible.

\subsection{Review of Past Work}

Boody [5] created a Quine McCluskey Calculator launched on PlayStore in 2012 using Java implementation of the algorithm. Boody [5] implementation allowed users to calculate for don't care values and showed the "Prime Implicants" (PI) charts as they were processed. However the version was limited to 4 variables and focused only on preparing the Karnaugh map and final result. It did not have the functionality of visualizing the resulting circuit diagram at a fast refresh rate.

Cits [6] developed a Karnaugh Veitch Diagram (KVD) calculator which calculated up to five variables based on Quine McCluskey implementation.KVD calculator displays the PI chart calculation and has a remarkable refresh rate between calculations. İts results were the most accurate of all the programs sampled for this review, although it does not generate circuit diagrams. Abdelrahman [7] developed an implementation of Karnaugh map minimizer for infinite variables using $\mathrm{C}++$. The $\mathrm{C}++$ code is relatively compact but does not have an explicit user interface which is an obvious disadvantage for achieving real time visual learning. Although the implementation gives the user the ability to input an infinite number of variables for calculation, processing limits is dependent on the power of the computer's processor.

GatePlus developed in this work provides a well presented, simple to use simulator for Boolean logic operations for up to six variables. It provides a tool for simple computation and graphical visualization of the results of logic functions and their corresponding circuit diagrams. Users will be able to easily see the results of any modifications they make, or any new methods they wish to employ. This tool will effectively make the study of Boolean logic functions easier, more interactive and practical.

\subsection{Objectives of Study}

The aim of this work was to design and develop a simple-to-use application for the simplification of Boolean logic expressions which will be optimized for User experience (UX) and User Interface (UI) design. The objective was to create a learning tool which assists both students and their teachers without the need for manual calculations to:

(i) easily and effectively simulate simple and complex Boolean equations,

(ii) visualize both the simplified logic equation and the Karnaugh maps solution

(iii) visualize in real-time circuits resulting from their design specifications, so that they can fine-tune and apply corrections in real time.

\section{MATERİAL AND METHODS}

The design used Unity3D development environment which provided an interface for the design of the front end of the software. Details on the Unity3D are found in [8]. Its development environment was specifically designed to handle highly flexible $2 \mathrm{D}$ and $3 \mathrm{D}$ graphics in Cartesian virtual space, but most of those tools are used in improvisation for the building of the graphical user interface of the software.

A custom built version of Javascript (Unityscript) was used to write the back end code that drives the algorithm. JavaScript code works at the back end for driving the dissolution of redundancy in the raw output from the truth table using the Quine-McCkluskey algorithm. The software provides an interactive user interface with three scrollable panels displaying:

(i) the truth table with a maximum of six inputs

(ii) the Karnaugh Map as well as the generated corresponding simplified logic equation.

(iii) the generated logic circuit diagram.

The logic conditions can be varied either on the truth table or on the Karnaugh Map panels while the logic equations and circuit diagrams of the simplified 
expressions are automatically generated from the output parameters decided on by the user for either sum of product (SOP) or product of sum (POS) approaches. The software tool is capable of working on multiple solutions on different tabs at the same time. Fig 1 shows the Unity3D development environment with GatePlus in active development.

\subsection{Project Mockup}

The drafting of a mock-up for the interface of the software can be done in any graphics compositing software. Adobe Photoshop was chosen for its flexibility. Before the digital drafting, it is advisable to analyse the optimal configuration for the software interface to deliver all the information to the user in the most minimal way, without cluttering the screen. This can easily be resolved by understanding the required functionality and drafting the cardinal functions. For GatePlus, these functions are:

i. The display of all possible combinations of input states for all current input variables, and the resulting output for that configuration. This functionality clearly requires the need of a Truth Table within the software interface. It will be preferable if this element is non-collapsible as it displays critical information that may need to be visible at all times.

ii. The visualization of the active circling of variable interpretations within a Karnaugh Map, so the user can see the graphical resolution of the equation generated from the truth table. The user must also be able to edit the output directly from the k-map, so as to be able to visually alter the outcomes of the truth table to simulate theories the user might have, or physically refine the Boolean relationship to generate a more favourable result.

iii. The software program must be capable of automatically generating a Logic Circuit Diagram based on the final solution. This element will require a lot of screen real-estate and needs also to be non-collapsible as the essence of the entire project is that the user should be able to at all times, visualize the dynamics in the nature and size of the circuit while altering ouput requirements.

iv. The software must have the functionality of toggling between Sum Of Product (SOP) and Product Of Sum (POS) calculations for output states. When SOP is selected, the on-state variables are active while the off-state variables are inactive, and vice-versa for POS calculations. This brief analysis shows that the most flexible configuration for the software would be to have three major panels. These panels will need to be enclosed in scrollable spaces to improve the software's screen real-estate management. Depending on the complexity of the solution being handled per time, the size of the enclosed scroll spaces will change (expand or contract) to accommodate the data being handled. This will be handled by unity's BeginScrol/View() and EndScrollView() classes.

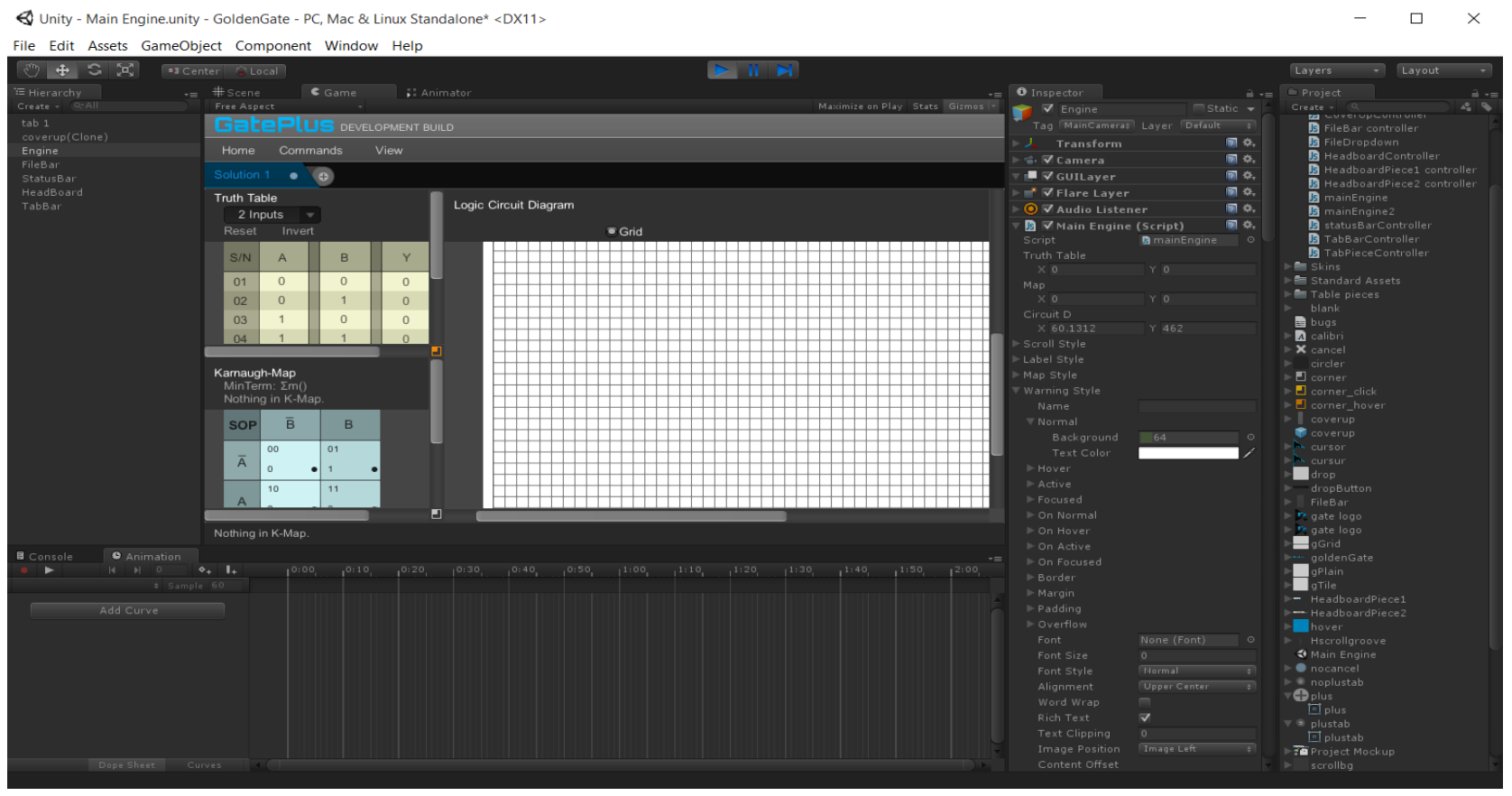

Figure 1: Unity3D development environment with GatePlus in active development. 


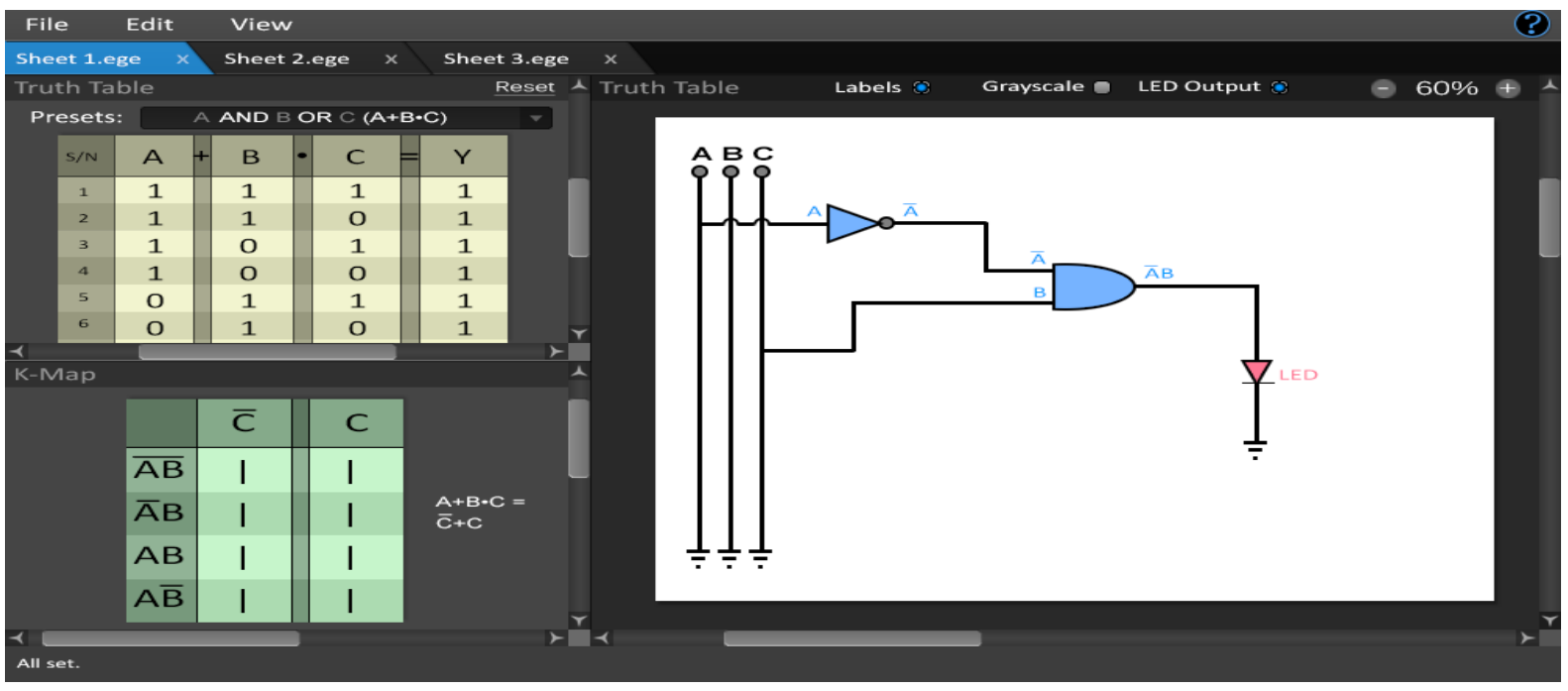

Figure 2: Mock-up for software interface of GatePlus.

Each scrollable space will also have a panel above it for simple configurations and information display. For instance, the title bar of the Truth Table will have options for choosing the number of variables, inverting the output (useful for resetting the table to no output immediately after a POS-SOP switch), resetting the solution or choosing from a list of preset solutions. The Logic Circuit Diagram title bar will also have options for toggling colour and grid on or off. Figure 2 shows the original project mockup for GatePlus 1.0.

Although the project Mockup of Figure 2 gives the developer a great deal of advantage in understanding the final product he is working towards, it has its limitations. As the project is mostly unstarted when the Mockup is designed, the developer might be unaware of some intrinsic details related to what might be required to be displayed on the interface, or what problems may arise in implementing some of his decisions. In retrospect to having completed the development of the software program, below are a few elements of the final application that did not appear in the Mockup.

(i) The addition of inverting gates (NOT gates) to each input line of the Logic Circuit Diagram to ease processing involved in the otherwise cumbersome method of using "symbology" to represent nonintersection between input lines when they are crossed, and channelling the input into the inverting gate right before it goes into the first layer of logic. Prior to the design, the added computing required to calculate for the small but vastly repeating non-intersecting elements in the circuit were not put into consideration.

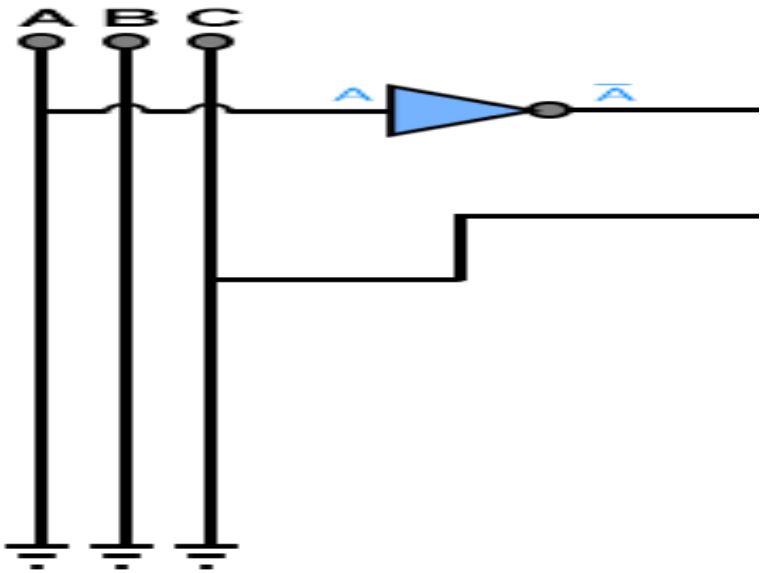

Figure 3: Mockup concept of variable roots in circuit diagram.

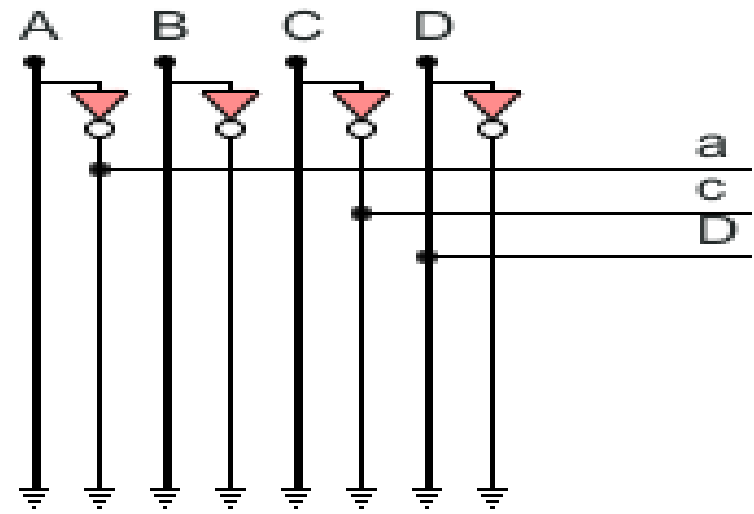

Figure 4: Actual implementation in final software design.

In the final actualization, two tiny buttons were added to the edge of each scrollable panel, between the vertical and horizontal scrollbars, and at border intersection points, to help the user navigate quickly to the top-left and bottom-right corners of the 
scrollable space when working with large spaces during more complex calculations. This was not considered in the conceptualization phase.

(ii) In the chosen "symbology", capital roman alphabets were used to represent input variables, with horizontal accent bars symbolizing an offstate or null value. In the actual implementation, plain capital roman alphabets were used to represent on-states, while small letters were used to represent off states. This is conventional in software implementations of Karnaugh maps, and was also utilized in this project to simplify programming and to reduce the processing involved in appending the accent bars to each off-state element in the map and circuit.

(iii) Two labels displaying grey-code and zeroindexed positions of each block in the Karnaughmap were added during active development.

(iv) A simple button for the addition of new tabs was also omitted in the project Mockup.

\subsection{Project Checklists}

This subsection describes the checklisting of key objectives that will need to be implemented for the software to execute its functions optimally. Each checklist serves as a landmark in the development process and systematically guides the design of the software to a desired finish. Below are the cardinal objectives that had to be implemented serially for the software to execute properly.

\subsubsection{Variable definition}

The mainEngine.js script that contains most of the code that drives GatePlus contains over 200 variable definitions for Arrays, lists, integers, doubles, textures, GUI elements, vectors, Booleans and strings. One cannot possibly know every single variable necessary to develop the software from the start. Variables must constantly be created during the development process to fulfil constituent tasks as they arise.

\subsubsection{Easy selection of number of variables to work with.}

Users can work with between 2 and 6 input variables: This was implemented using a single integer variable labelled internally as no Of Parameters with a default value of 2 .

\subsubsection{Automatically formatted truth table with editable output variables}

This is one of GatePlus's more robust features. The truth table panel is nested within a Scrollable space implemented by calling the inbuilt function, GUI. BeginScrollView () from within the OnGUI () function, the cardinal function that controls the display of all GUI elements in Unity. GUI. Draw Texture () is used to create the visual elements of the table while a complex chain of loops helps to generate and control the dynamics of the binary numbers that express the possible permutations of configurations of input variable states, as well as the dynamics of the table's form as the number of variables (no Of Parameters) changes. Finally, the output values toggle from onstate to off-state by the use of invisible buttons behind the text, with a Boolean control.

\subsubsection{Editable, realtime, self-solving Karnaugh map}

This is another robust feature of GatePlus, whose intricate details cannot possibly be explained in its entirety in one subsection. The script is nested in a similar manner as that explained above for the truth table. The circling of PIs is controlled by a reverseengineering process due to the complexities involved in programming a linear system to compute the torus-shaped continuity characteristics of the Karnaugh map. The reverse-engineering process is realized by writing a custom function to interpret the linear results from the Quine-McCluskey algorithm to circled groups, and apply the correct outline at the right position, to the Karnaugh map. The circle shapes are colour-coded to aid their visibility: green for unit or square groups, red for vertical groups, yellow for horizontal groups and blue for groups that circle virtually due to torus characteristics, but not always physically.

\subsubsection{Easy toggling}

Between Product of Sum (POS) and Sum of Product (SOP) states: This is realized using a single button on the top-left corner of the Karnaughmap. About three if-loops running in the update function tap out of the Boolean output of this function to ensure that its results are translated to all elements of the software that need to be altered when the switch is toggled. These elements include (but are not limited to) toggling the string stating the Boolean parameters from min to max terms or vice-versa, toggling the 
values to be considered from the truth table to offstate variables in the case of POS or on-state for SOP variables and expressing the simplified Boolean expression in the appropriate form.

\subsubsection{Switch for inversion of output variables} for sake of ease of implementation of same results from SOP to POS and vice versa without having to start afresh. This is implemented in a single custom function called invert() which is called whenever one of the invert buttons on the interface is clicked.

\subsubsection{Switch for resetting output variables to} their default values in POS or SOP form. This is implemented in a single custom function called reset() which is called whenever one of the reset butons on the interface is clicked.

2.2.8 Dynamic circuit diagram (adjusts itself automatically to display the results of the Karnaugh map).

\subsubsection{Multitasking capabilities: Multiple} "renamable" tabs can be opened to handle several calculations per time. Users can work with between 1 and 5 renamable tabs.

2.2.10 Professional software interface: The software must meet professional standards for presentation and user experience. The interface design is themed after Adobe Photoshop CS6. Several functions and design specifications are implemented to make the software meet the required standards namely:

a. Smooth-scroll buttons: To take the scrollspace directly to the top or bottom with the click of a simple button.

b. Guiding animations: The tabs slide out to show that a new tab has been created, or slide in to show that a tab has been destroyed.

c. Colour signalling: The colours of certain icons or elements change to show that they are in focus or that your attention is needed (e.g. blue for focus, and grey for latency on the tab buttons). This is especially used in the Karnaugh map, to indicate the size and scope of circled regions, or demarcations for laterally inverted zones.
2.2.11 Status Bar: To display relevant information, as well as solutions to problems being solved at that particular session.

2.2.12 Menu Bar: To provide a comprehensive list of software related options.

\subsection{Main Framework of the Driving Code for the Application's Functionality}

Unity Script works by having very different execution behaviours for different parts of the script. Below are descriptions of the execution behaviours of three major functions that are extremely relevant to the execution of this project.

\subsubsection{Function Mono Behaviour. Start (); void}

\{\}: This function runs anything enclosed within its curly brackets only once-when the application is started. This means it is useful for one-time execution of portions of the code that need only be executed once, like setting initial states for some variables (mostly JavaScript arrays). The empty brackets indicate that this function accepts no input variables when it is called and returns nothing hence the "void" after the colon (Note that in Unity Script, the scripting convention does not permit the actual addition of ": void" when the function is called. This is mentioned only for emphasis for the sake of this description).

\subsubsection{Function Mono Behaviour. Update ():} void \{ $\{$ : This function runs in a constant loop, on every frame. A frame is a video-based subdivision of time during which static elements are displayed on the screen. Elements change only when a frame changes, thus creating the illusion of motion. Unity's standard is 60FPS (Frames Per Second), so the update function runs its enclosed script from top to bottom, sixty times every second. Most of the timedependent calculations in the code are executed from within this function. The function's unique running behaviour can also be adopted on other functions by calling them from within this function.

\subsubsection{Function Mono Behaviour. On GUI():} void \{\}$:$ All operations directly related to the display of two dimensional graphical elements like labels and buttons (Virtually the entire interface of the software) can only from run within this function. This is due to the peculiar way that GUI elements are processed by the computer. Depending on the power of the graphics card and the processor speed, computers 
need to have a high refresh rate for the elements on the screen, as they are being re-rendered constantly so changes can be seen immediately by the user once they are executed from the back-end. This is different from the frame rate because it is processor dependent and may run at close to twice the speed of the Update function. For this purpose, the running behaviour of the Update() and OnGUI() functions had to be considered very carefully during the development process, especially when they needed to synchronise to produce a desired output. Fig 5 shows: Mono Develop 3.0 interface with simple script showing major functions.

\section{TESTS, RESULTS AND DİSİCUSSİON}

The testing criteria focused on are start-up speed, hardware performance, and accuracy of results obtained from the software. Unit tests are executed using Unity's inbuilt profiler, which offers detailed diagnostics containing details of the software's performance while running.

The software is tested on full performance by using intermittent $\mathrm{ON}$-states in the truth table as this configuration ensures that there is no redundancy, thus forcing the script to run the highest number of loops. The checked pattern and green outlines on the ON-states in the Karnaugh map, as well as the large number of AND gates in the logic circuit diagram further increase loading since the software has to redraw a much larger number of GUI elements for every frame.
Tests were carried out on a HP Envy 14 with a $2.2 \mathrm{GHz}$ Intel Core i5-5200U quad core processor (overclock to $2.7 \mathrm{GHz}$ ), Intel HD 5500 graphics, 1TB HDD and 12GB of RAM, running Windows 10 on a $1920 \times 1080 p$, $60 \mathrm{~Hz}$ display. Figure 6 and 7 show GatePlus highest loading configuration for four variables for both truth Table and Circuit diagram respectively.

\subsection{Start-up Speed Test}

On the testing system described in the previous section, start-up duration of the core engine is between $100 \mathrm{~ms}$ and 3s, depending on system's processing load at the time of execution. However the entire package executes about 5-8 seconds slower because of a deliberately timed delay to allow for the display of the software's start-up splash screen shown in Figure 8.

\subsection{Hardware Impact}

Ten samples for CPU, Memory, and Disk performance impact were recorded from the system performance analyser within the windows task manager and the average taken. Readings were taken on the high performance power profile and with no other applications open. The results for the application in idle state and in full load are recorded in the table 1 for 2-6 variables. Notice the large rise in CPU usage from 5 variables full load and upwards. However memory and disk usage did not change appreciably as number of variables increased.

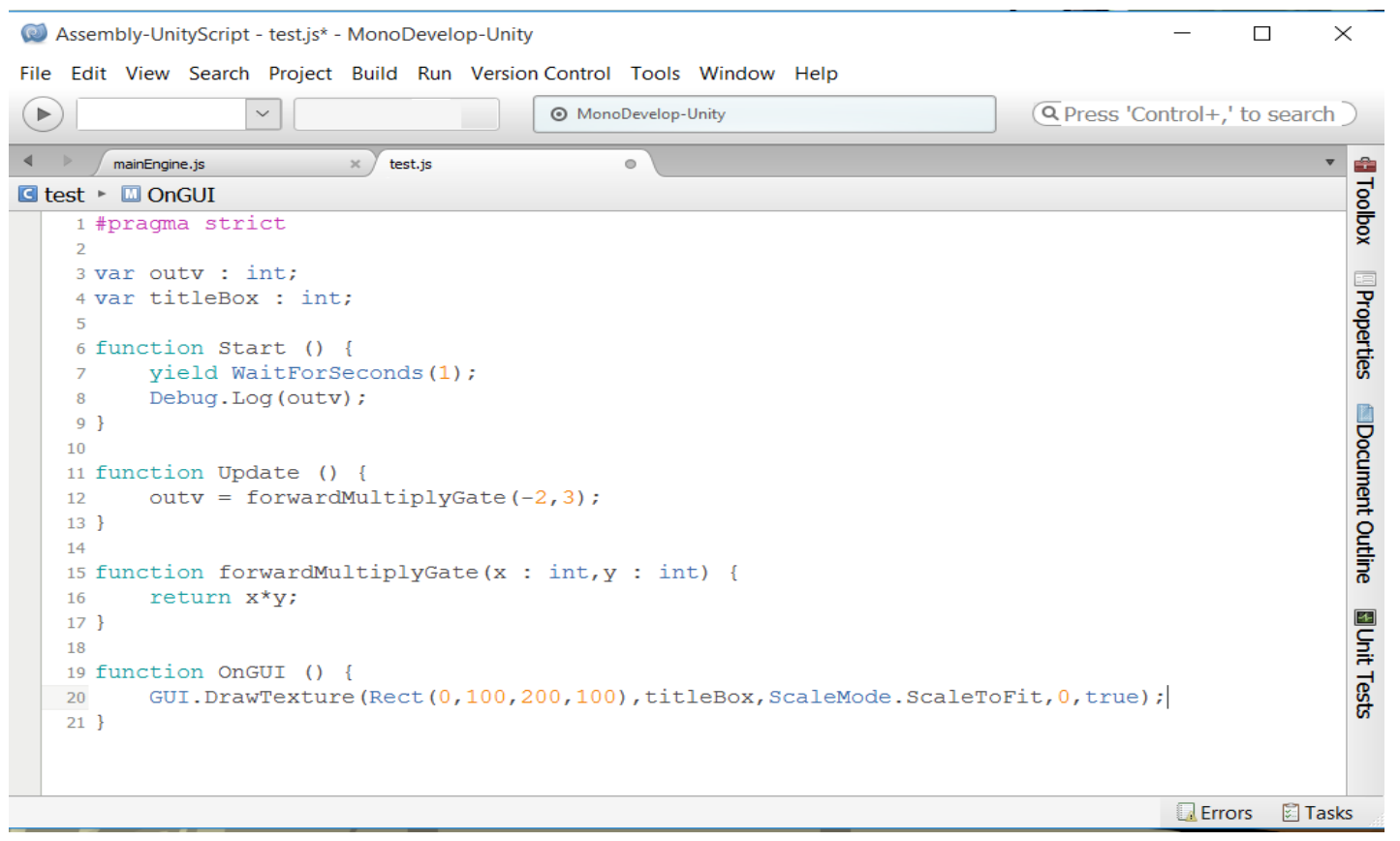

Figure 5: Mono Develop 3.0 interface with simple script showing major functions. 


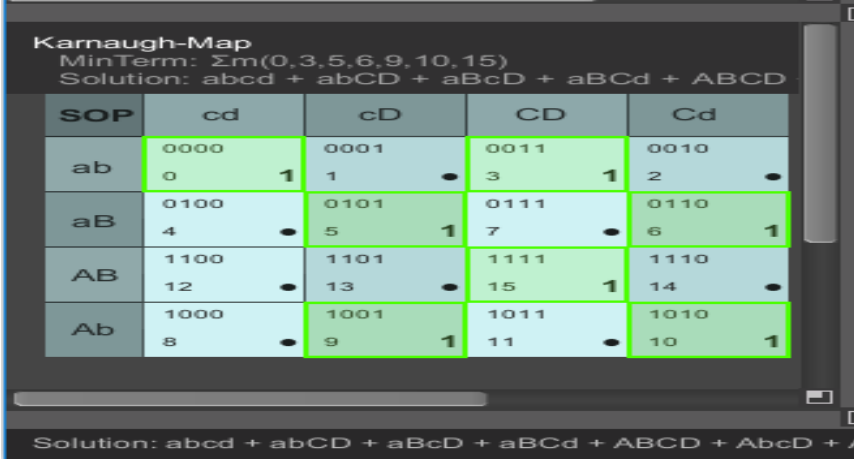

Figure 6: GatePlus Truth Table highest loading configuration for four variables

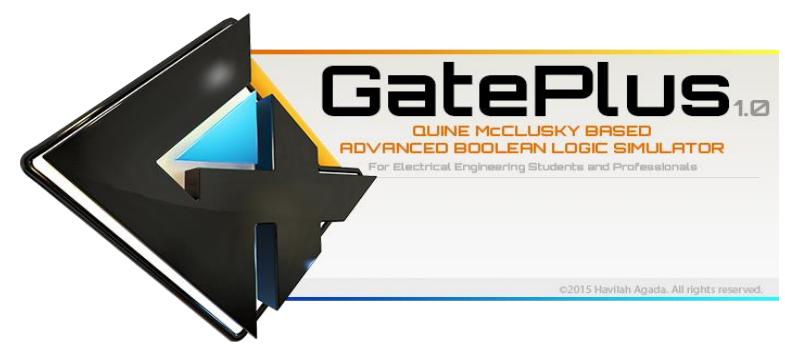

Figure 8: GatePlus 1.0 splashscreen.

Figure 9 shows Unity profiler monitoring GatePlus's performance on 6 variables with full load. The Truth Table, Karnaugh map, logic equation and Circuit diagrams provided by GatePlus are also shown in the diagram.

Table 1: Hardware Performance of GatePlus

\begin{tabular}{ccccc}
\hline S/N & State & CPU (\%) & Memory(MB) & Disk(MB) \\
\hline 1 & 2 Variables, Idle & 8.6 & 48.3 & 0.0 \\
2 & 2 Variables, Full & 8.7 & 48.5 & 0.1 \\
3 & 3 Variables, Idle & 10.3 & 48.5 & 0.0 \\
4 & 3 Variables, Full & 12.4 & 45.8 & 0.2 \\
5 & 4 Variables, Idle & 12.9 & 48.9 & 0.0 \\
6 & 4 Variables, Full & 13.3 & 49.0 & 0.1 \\
7 & 5 Variables, Idle & 18.3 & 49.4 & 0.0 \\
8 & 5 Variables, Full & 33.8 & 49.6 & 0.2 \\
9 & 6 Variables, Idle & 30.3 & 50.2 & 0.2 \\
10 & 6 Variables, Full & 30.4 & 50.3 & 0.2 \\
\hline
\end{tabular}

Any change effected by the user on the truth Table or the Karnaugh map is automatically reflected in real time in the Boolean logic equation and the circuit diagram. Notice the sharp rise in CPU processing for 5 variables full and above on Table 1, hence the need for the warning pop-up shown in Figure 9 which was expanded in Figure 10.

\subsection{Speed and Accuracy Test}

GatePlus was tested against hand solved problems and other logic solvers, and was found to be completely

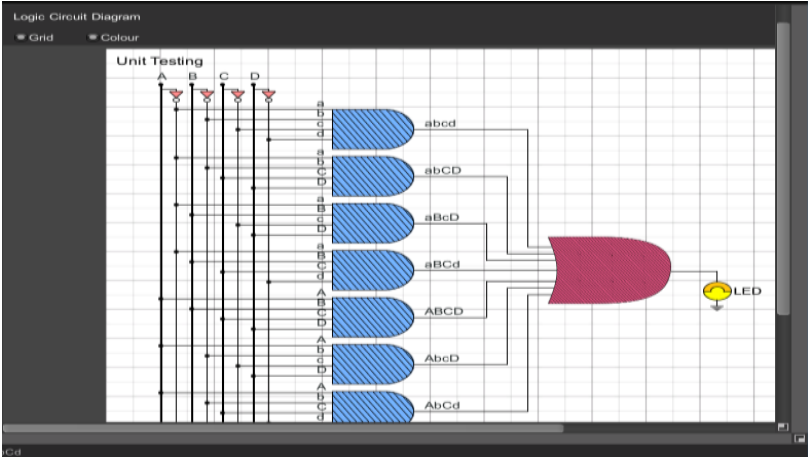

Figure 7: GatePlus Circuit Diagram highest loading configuration for four variables.

accurate in both Product of Sum and Sum of Product implementations. There is absolutely no noticeable delay between computation changes due to the use of the update function to call the Quine-McCluskey solver, and the building of the other essential panels directly from the results of the continuously synchronizing solution. However it should be noted that the simulator was tested on a relatively fast CPU and a great deal of RAM, so performance may differ slightly on a different platform.

The results of the simplified Boolean expressions and logic circuits generated by the developed tool were compared with manually calculated solutions and available html and console based implementations and were found to be accurate and with a better user friendly graphic User Interface $[9,10]$.

\subsection{Bugs}

Below is a list of recorded bugs in GatePlus 1.0

i. The drop-down list for number of variables may not automatically exit the screen when the user clicks away from it, as in standard software.

ii. Once the quick-scroll corner-button for the Logic Circuit scroll space is clicked once before the scroll space is large enough to require a vertical scrollbar, the horizontal scrollbar will continue to orient itself to the right even when pulled away. This will continue until the vertical scrollbar appears. This is due to conflict in an exponential value controlling its animation, and the screen.length read-only variable. However it does not at all hinder performance or distract the user.

iii. There are a few pixel-position-related inconsistencies with the lines in the Logic Circuit diagram when some parameters are passed to it. The diagram is although, still readable, but may not display conventionally. 
iv. In extremely rare occasions, the Karnaugh map may not circle a Prime Implicant displayed on the Karnaugh map. It is important to note that this is a purely visual error from the graphics processing and not the Boolean logic simulator engine. The simplified Boolean logic solution and the Logic Circuit diagram will still be correct. Just make sure to check the status bar and Karnaugh map header bar to be sure.

\section{FUTURE RESEARCH DIRECTION}

Several improvements can be implemented in a future build of the software. Listed below are a few of the features that will need to be added to what has already been done:

(i) Addition of more than 6 inputs for calculation.

(ii) NOR and NAND gate implementation in the Karnaugh map. (iii) Functionality to export circuit diagrams and Karnaugh maps as image files for printing and reference outside the software.

(iv) Addition of panel to view Quine-McCluskey primeimplicant charts during computation.

(v) Creation of tutorial mode to more interactively guide new users

\section{CONCLUSION}

The development of a fully functioning Boolean logic simulator using the Quine-McCluskey algorithm was carried out successfully. Its results are accurate with seamless automatic synchronization. The overall size of the software package is about 50MB, a considerable file size. The procedure was however not without challenges. Ideally, the algorithm utilized can be computed to an infinite number of variables but due to the constraints of computational speed and complexity, only six levels were implemented in the software.

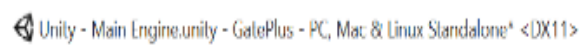

File Edit Assets Gameobject Component Visual Sudio Tools Window Help

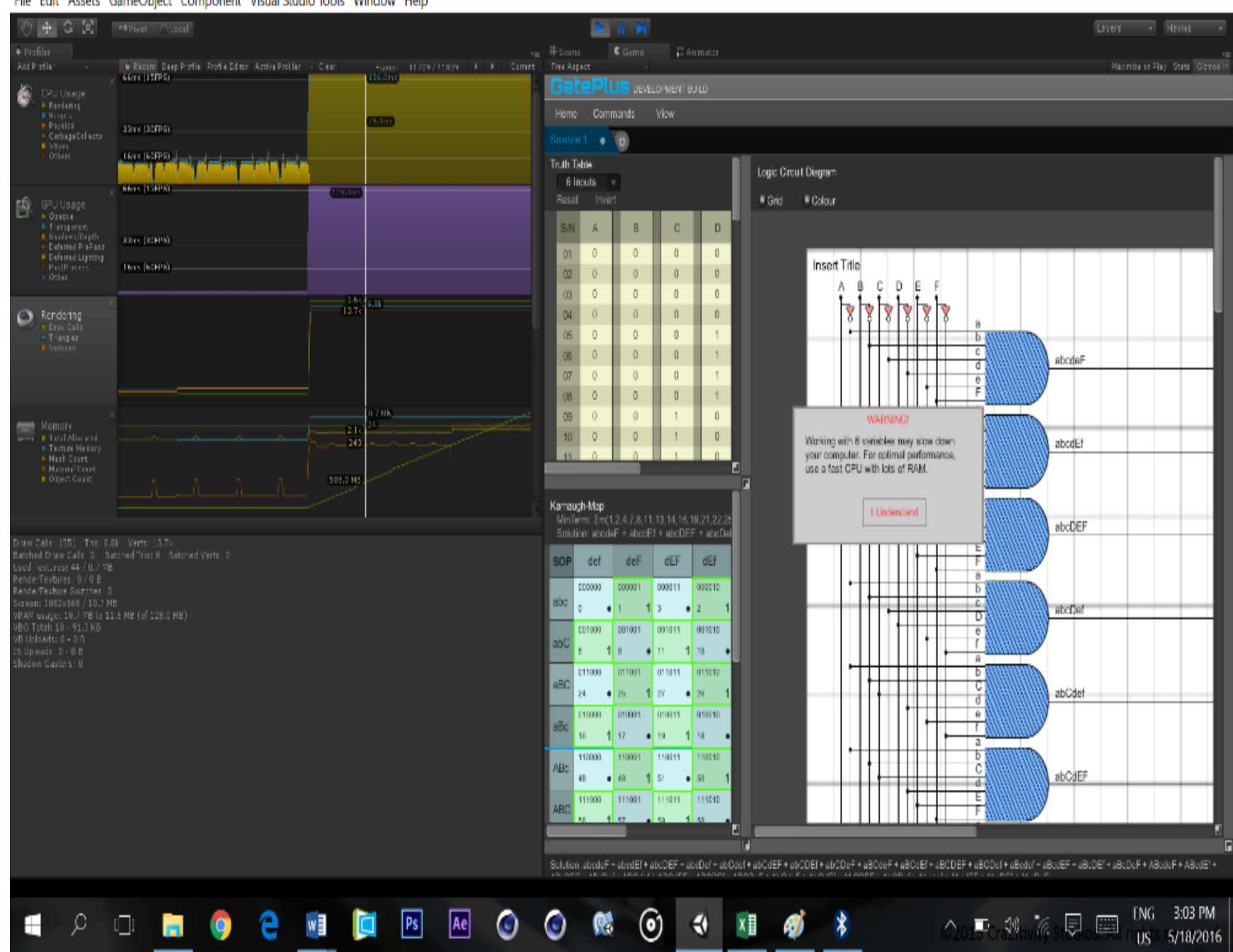

Figure 9: Unity profiler Monitoring GatePlus's Performance on 6 Variables with Full Load. 


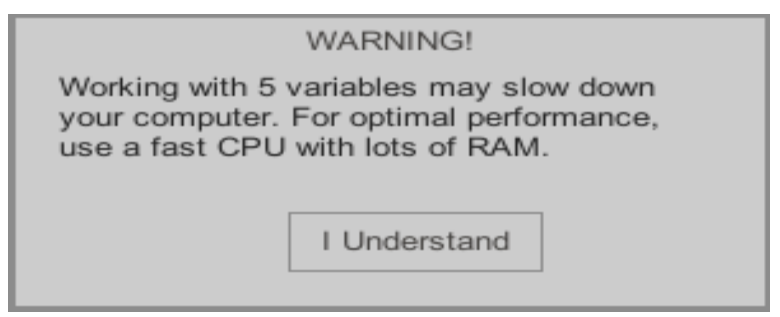

Figure 10: Warning pop-up when working above 4 variables.

This software can be utilized by students and professionals for learning purposes and for simple Boolean simplification for projects they may be working on. It is easy to use and can be learnt in less than ten minutes. To the best of our knowledge, the developed logic simulator tool has an edge over previous implementations $[6,7,8,11]$ because it provides a more robust GUI suitable for easy visualisation and multitasking needed for educational purposes.

\section{REFERENCES}

[1] Petrescu, I., Păvăloiu, I., \& Drăgoi, G.: Digital Logic Introduction Using FPGAs. Procedia - Social and Behavioral Sciences Vol.180, pp1507 - 1513. Elsevier (2015).

[2] Frank M. B.: On the Suppression of Variables in Boolean equations. Discrete Applied Mathematics. Vol. 159 pp255-258. Elsevier (2011).

[3] Gribomont, E. P.: Simplifications of Boolean verification conditions. Theoretical Computer Science. Vol.239 pp165-185. Elsevier (2000).

[4] Thomas J. Murray \& Mohan R. Tanniru. Control of inconsistency and redundancy in PROLOG-type knowledge bases. Expert Systems with Applications, Volume 2, Issue 4, 1991, Pages 321331.
[5] Boody, J.: Quine-Mckluskey Calculator. Google Playstore 10/08/2016 at https://play.google.com/store/apps/details?id=lu abear.qm4android\&hl=en (2012).

[6] Cits.: Karnaugh Veitch Diagram Calculator. Google Playstore Retrieved 10/08/2016 at https://play.google.com/store/apps/details?id=co m.mhsoft.kvd\&hl=en (2013).

[7] Abdelrahman, E.: A C++ Karnaugh Map Minimizer-Infinite Variables. Code Project. Retrieved 10/08/2016 at http://www. codeproject.com/Articles/649849/A-CplusplusKarnaugh-Map-Minimizer-Infinite-Variab (2014).

[8] Sometimes I Code.: How does Unity3D Scripting work under the hood? Wordpress.com. Retrieved 9/08/2016 at https://sometimesicode .wordpress.com/2014/12/22/how-does-unitywork-under-the-hood/ (2014).

[9] Huang, J.: Programming implementation of the Quine-McCluskey method for minimization of Boolean expression. Department of Biological Sciences, Faculty of Science, National University of Singapore. Retrieved 29/05/16 at https://arxiv. org/ftp/arxiv/papers/1410/1410.1059.pdf(2014).

[10] Habib, A. H. M. A., Salam, M. A., Nadir, Z. \& Hemen G.: A new approach to simplifying Boolean Functions. The Journal of Engineering Research, Vol. 1 pp39-45. (2004).

[11] Muhammed M. O.: Karnaugh Map Minimizer (Three Variables). Code Project. Retrieved 10/08/2016 at http://www.codeproject.com/ Articles /37031/Karnaugh-Map-Minimizer-ThreeVariables (2013). 